\title{
Making a splash with splicing
}

Cell Research (2017) 27:457-458. doi:10.1038/cr.2017.24; published online 24 February 2017

In a recent Nature paper, Heintz et al. identify a splicing factor (SFA1) that is crucial for the longevity conferred by dietary restriction and the TORC1 pathway modulation in C. elegans.

Dietary restriction (DR) is one of the most reliable and best-studied ways to increase lifespan experimentally. Its effects, however, like all physiological processes, depend on the exact genotype of the experimental organism [1]. Furthermore, different DR regimens act by distinct as well as by overlapping genetic mechanisms [2]. Thus, by identifying common mechanisms, it might be possible to develop interventions that mimic the beneficial effects of DR. The much studied TORC1 (target of rapamycin complex 1) pathway controls responses to nutrient availability and its inhibition promotes longevity. In a recent paper in Nature, Heintz and colleagues uncover a new link between the TORC1 pathway and DR in C. elegans by identifying a splicing factor that is required for the longevity conferred by two different DR regimens as well as by TORC1 pathway modulation [3].

Using an in vivo fluorescent alternative splicing reporter, Heintz and colleagues show that splicing is under tight regulation during development, however, like much else in the cell, becomes deregulated during aging. They observe that during development, the splicing reporter undergoes stereotyped changes in alternative splicing. However, by day 5 of adulthood, there are changes in exon usage that vary between tissues and individuals, with an increased incidence of exon skipping. These changes are predictive of subsequent lifespan.
Indeed, when animals are sorted into groups based only on their patterns of exon usage early in adulthood, the group with increased exon skipping lived significantly shorter.

Here comes the twist: the authors found that a solid-plate dietary restriction (sDR) regime in which animals are fed bacteria at a lower concentration significantly increases lifespan, and leads to maintenance of splicing robustness. Using the fluorescent splicing reporter, they showed that compared to animals fed ad libitum, the sDR animals maintain a "youthful" pattern of splicing, that is consistent from animal to animal. Using RNA-seq, the authors observed a global deregulation of mRNA processing with age. Aged animals showed a significant increase in intron retention and use of atypical splice junctions compared to younger animals. sDR slowed down the process. sDR animals on Day 15 did not show any increase in aberrant splicing compared to younger animals. However, by Day 27, sDR animals exhibited a significant increase in aberrant splicing, similar to animals fed ad libitum on Day 15.

To identify spliceosome components required for DR-mediated longevity, Heintz et al. screened conserved splicing factors to identify those that suppress the increased lifespan of the eat-2(ad1116) mutant, a much used genetic model of DR [4]. They identified the branch-point binding protein splicing factor 1 (SFA-1). Knockdown of $s f a-1$ supressed the increased lifespan conferred by eat- 2 as well as by sDR. Knockdown of $s f a-1$ also restored the age-dependent changes in splicing that are prevented by DR, that is, DR no longer protects from aberrant splicing in the absence of $s f a-1$. Moreover, a known target of SFA-1, target of splicing (tos1), showed age-dependent changes in splicing that are prevented by eat 2 . The effect of eat-2 on tos- 1 splicing was suppressed by $s f a-1$ RNAi. Together, these data suggest that it is only when SFA-1 is intact that DR can slow down age-dependent changes in splicing and aging itself.

Is SFA-1 required for other genetic interventions that increase lifespan? In C. elegans, in addition to DR, two other well-established means for increasing lifespan are via altering insulin-like signaling (such as in daf-2 mutants) and via modulation of the mitochondrial electron transport chain. Heintz et al. showed that SFA-1 is not required for the increased longevity of daf-2(e1370) mutants. However, it is required for the longevity of isp-1(qm150) mutants, which have a mutation in the Rieske iron-sulfur protein of Complex III [5]. Some mechanistic overlap between mitochondrial respiratory defects and DR may indeed be logical as both must affect general metabolic functions.

One difficulty with many genetic studies of aging is that when one observes that a particular mutation or treatment suppresses a long-lived mutant, one cannot easily distinguish between the two possible ways it could be preventing lifespan extension. Does the intervention inhibit the core of the pro-longevity mechanism or does it simply affect a permissive activity that is necessary for longevity in that particular context. For example, increased reactive oxygen species (ROS) generation in isp-1 mutants actively promotes longevity and is suffi- 
cient for lifespan extension [6]. Whereas an intact mitochondrial unfolded protein response (mtUPR) is only permissive: it is required for the longevity of $i s p-1$ mutants, but is not sufficient [7]. Heintz et al. addressed this issue head on in their study by examining whether SFA-1 activity is by itself sufficient to increase lifespan. Indeed, the authors observed a moderate lifespan increase by overexpression of $s f a-1$. This finding suggests that changes in splicing can actively promote pro-longevity mechanisms, and therefore may be a promising line of inquiry for future aging studies.

Finally, how is DR mechanistically linked to splicing? Components of the TORC1 pathway have previously been shown to be mediators of the beneficial effects of DR [8]. Here, Heintz et al. identify a TORC1 pathway component, RAGA-1, as a crucial link between DR and splicing. Loss of raga-1 blocks age-dependent splicing of the SFA-1 target tos- 1 and abolishes the effect of sDR on age-dependent splicing of the fluorescent reporter. The link between splicing and the TOR pathway is further strengthened by the finding that, similar to its effect on DR, loss of $s f a-1$ suppresses the lifespan extension conferred by genetic manipulations which decrease TORC1 signaling, such as loss of the TORC1 activator RAGA-1, loss of the TORC1 effector RSKS-1/S6 kinase, and constitutive activation of the TORC1 inhibitor AAK-2/AMP-activated protein kinase. It is likely that these very interesting relationships between DR, splicing and TORC1 signaling will keep many good minds occupied for some time to come.

Robyn Branicky ${ }^{1}$, Siegfried Hekimi ${ }^{1}$

${ }^{\prime}$ Department of Biology, McGill University, Montreal, Quebec, Canada H3A $1 B 1$
Correspondence: Siegfried Hekimi

E-mail: siegfried.hekimi@mcgill.ca

\section{References}

1 Liao CY, Rikke BA, Johnson TE, et al. Aging Cell 2010; 9:92-95.

2 Greer EL, Brunet A. Aging Cell 2009; 8:113-127.

3 Heintz C, Doktor TK, Lanjuin A, et al. $\mathrm{Na}$ ture 2017; 541:102-106.

4 Lakowski B, Hekimi S. Proc Natl Acad Sci USA 1998; 95:13091-13096.

5 Feng J, Bussiere F, Hekimi S. Dev Cell 2001; 1:633-644.

6 Yang W, Hekimi S. PLoS Biol 2010; 8:e1000556.

7 Wang Y, Hekimi S. Science 2015; 350:12041207.

8 Hansen M, Taubert S, Crawford D, et al. Aging Cell 2007; 6:95-110. 